\title{
Amateur Astronomers and the Hubble Space Telescope
}

\author{
Stephen J. Edberg \\ HST Amateur Astronomers Working Group, 163 Starlight Crest Dr., \\ La Cañada, CA91011, U.S.A.
}

\section{Background}

In December 1985 the leaders of major amateur astronomy organizations in the U.S. met with the director and staff of the Space Telescope Science Institute (STScI) at their invitation to discuss the possibility of organizing a program for U.S. citizens who don't work professionally in astronomy to make use of the Hubble Space Telescope. Director Riccardo Giaconni's previous successful experience in cooperating with the American Association of Variable Star Observers (AAVSO) on studies of various objects with the orbiting High Energy Astronomy Observatory prompted him to bring together representative of the AAVSO, Association of Lunar and Planetary Observers (ALPO), Astronomical League (AL), International AmateurProfessional Photoelectric Photometry (IAPPP), Independent Space Research Group (ISRG), International Occultation Timing Association (IOTA), and Western Amateur Astronomers (WAA) to work out an approach to making HST observing time available to amateur astronomers, educators, and anyone else who might have a good research idea. Dr Giaconni offered to make some of his director's discretionary time available to qualified non-professional astronomers if the representatives present would design and carry out a program to find the good research ideas and have them ready for his selection. They would then be inserted in the HST observing program once the first six months of Guaranteed Time Observations had been carried out and the regular General Observing program had begun. With this as its charge, the representatives organized themselves into the Amateur Astronomers Working Group and have prepared a procedure for the selection of non-professional researchers to use the Hubble Space Telescope.

\section{The Program}

Certain restrictions were imposed on the AAWG by the National Aeronautics and Space Administration (NASA) and the STScI at the outset:

1. The proposed study must make use of the HST's unique capabilities, and must be impossible to do from the ground. These capabilities include its high resolution, extended wavelength range, and high sensitivity. 
2. The proposer must not be a professional astronomer: the AAWG defined a professional as one with an advanced degree in astronomy or astrophysics or one who draws salary doing or assisting in research or data processing in these fields.

3. The principal proposer must be a U.S. citizen though co-investigators need not be.

The AAWG has designed a multi-step process to send proposals to Director Giaconni. First, an initial 250-word essay describing the proposed research is submitted to the amateur organization most familiar with that area of research. Proposal forms and a handbook (Littmann, 1986) describing the HST were distributed for this purpose. Proposers passing this initial screening by the organization are then asked to do a more formal, detailed proposal and justification, which includes some of the standard STScI proposal forms distributed to the professional astronomical community. Each organization will again screen these proposals and bring the best to a meeting of the AAWG in which all the proposals at the meeting will be prioritized. A formal STScI observing request will be prepared for each and then the list will be submitted to the Director for his selection of one or more for scheduling on the HST. When the observations are to be made the successful proposer will be invited to STScI to monitor the acquisition of the data and perform analysis on them. Ultimately the results are to be published in an appropriate journal and a report submitted to the sponsoring amateur organization and to the STScI.

It is the hope of the AAWG that a successful program on the Hubble Space Telescope will open the door to similar programs at major ground-based observatories.

\section{References}

Beaty, J. Kelly, 1985 May, "HST: Astronomy's Greatest Gambit", Sky and Telescope

Littmann, Mark, 1986 October, Hubble Space Telescope Handbook for Amateur Astronomers, Space Telescope Science Institute, Baltimore

Longair, Malcolm, 1985 April, "The Scientific Challenge of the Space Telescope", Sky and Telescope

Watson, Gary, 1986 January, "Building the Space Telescope's Optical System", Astronomy 